\title{
Numerical Approach in Superconductivity: An advanced study
}

\author{
V. A. Kizka 1
}

Kharkiv National University of Radio Electronics, Nauky Ave. 14, Kharkiv, 61166, Ukraine

V.N. Karazin Kharkiv National University, Kharkiv, 61022, Ukraine

Joint Institute for Nuclear Research, Dubna, 141980, Russia

\begin{abstract}
The dependence of the critical temperature $T_{c}$ of high-temperature superconductors of various families on their composition and structure is proposed. A clear dependence of the critical temperature of high-temperature superconductors (hydrides, Hg- and Y-based cuprates) on the serial number of the constituent elements, their valence and crystal lattice structure has been revealed. For Hg-based cuprates, it is shown that it is possible to obtain even higher temperatures of superconducting transitions at normal pressure by implanting mercury atoms into the crystal lattice of cuprate.
\end{abstract}

Keywords: High-temperature superconductivity; critical temperature.

\section{Introduction}

Recent results in the field of high-temperature superconductivity have aroused great interest of the scientific community to search for a theoretical explanation of the mechanism of the appearance of a superconducting state at temperatures close to room temperature [1], [2]. However, many theoretical questions remain unresolved, while the experiment approached room temperature at high pressures and halfway to room temperature at normal pressures.

Various numerical approaches have been used to describe superconductivity. For example, numerical approach in description of vortex nucleation in the Ginzburg-Landau model of superconductivity [3] and a numerical method of solving the mean-field self-consistent Bogoliubov de Gennes equations by expanding the Nambu Greens functions in terms of Chebyshev polynomials 4 which both require phenomenological assumptions or complex calculations on grid. All of them do not give a clear dependence of the observed parameters of a superconductor on its material composition and lattice structure. In the article [2], good agreement was obtained between the critical temperature of the superconducting transition and the parameters of high-temperature superconductors, but these parameters themselves require experimental measurements and have a complex dependence on the composition and structure of the sample, which is still questionable. This study uses the basic idea that there is a clear relationship between the content and structure of a superconductor and its critical superconducting transition temperature, expressed in integer and rational numbers, as is the case for the laws of quantum mechanics.

An attempt is made in this work to establish a relationship between the superconducting transition temperature $T_{c}$ of various families of high-temperature superconductors on the composition and structure of the superconductor material. For this, a numerical approach is used, similar to Balmer's work using number theory, which was only once applied

\footnotetext{
${ }^{1}$ valerii.kizka@nure.ua
} 


\begin{tabular}{|c|c|c|c|}
\hline $\begin{array}{c}\text { Composition: } \\
H g-m 2(n-1) n\end{array}$ & $\begin{array}{c}\text { experimental } \\
T_{c},[\text { source }]\end{array}$ & $\begin{array}{c}T_{c} \\
\text { by the formula }[1]\end{array}$ & $\begin{array}{c}T_{c} \\
\text { by the formula }[2]\end{array}$ \\
\hline$H g-1201$ & $97 K[8]$ & $95 K$ & $134 K$ \\
\hline$H g-1212$ & $128 K[8]$ & $128 K$ & $140 K$ \\
\hline$H g-1223$ & $135 K[8]$ & $132 K$ & $152 K$ \\
\hline$H g-1234$ & $127 K[8]$ & $120 K$ & $144 K$ \\
\hline$H g-1245$ & $110 K[8]$ & $108 K$ & $132 K$ \\
\hline$H g-1256$ & $107 K[8], 92 K[9]$ & $97 K$ & $121 K$ \\
\hline$H g-1267$ & $88 K[8]$ & $88 K$ & $111 K$ \\
\hline$H g-1278$ & $<90 K[8]$ & $80 K$ & $103 K$ \\
\hline$H g-2212$ & $44 K[8]$ & $47 K$ & $48 K$ \\
\hline$H g-2223$ & $45 K[8]$ & $51 K$ & $53 K$ \\
\hline$H g-2234$ & $114 K[8]$ & $48 K$ & - \\
\hline
\end{tabular}

Table 1: Table of experimental values of the critical temperature of cuprates based on $\mathrm{Hg}$ in comparison with those calculated by the formulas $(1-2)$.

to fundamental problems of physics over a hundred years ago when describing the emission spectra of hydrogen [5]. His result was confirmed by Rydberg [6] and explained by Bohr when he was creating quantum mechanics [7].

\section{Method and Results}

The essence of the applied method is as follows. Consider Hg-based cuprates $\mathrm{Hg}_{m} \mathrm{Ba}_{2} \mathrm{Ca}_{n-1} \mathrm{Cu}_{n} \mathrm{O}_{2 n+m+2}$ or another notation $\mathrm{Hg}-m 2(n-1) n$. Suppose that $T_{c}$ is proportional to the combination of integers, ordinal numbers of the elements of a given cuprate, and the number of elements in the chemical formula of the cuprate. Using the enumeration method (enumeration of various dependences of the critical temperature on the serial numbers of chemical elements of the substance), it turned out that the following combination is in good agreement with the experimental value of $T_{c}$ :

$$
\frac{1}{T_{c}}=\frac{C_{0} \cdot n \cdot m \cdot(m+1) / 2}{300 \cdot(2 N-3)^{2}}\left(\frac{1}{m \cdot H g}+\frac{1}{2 \cdot B a}+\frac{1}{(n-1) \cdot C a}+\frac{n}{n \cdot C u+O^{2 n+m+2}}\right),
$$

where $C_{0}=147.27\left[K^{-1}\right]$ is a fitting constant; $n$ and $m$ - integers from the material designation; the designation of an element is its ordinal number or the charge of the nucleus, i.e. $\mathrm{Hg}=80, \mathrm{Ba}=56, \mathrm{Ca}=20, \mathrm{Cu}=29, \mathrm{O}=8 ; \mathrm{N}$ is the number of elements, excluding $\mathrm{Cu}-\mathrm{O}$ : for example, $\mathrm{N}=2$ for $\mathrm{Hg}-1201$ (here two elements are mercury and barium), $\mathrm{N}=3$ for 1212 (here mercury, barium and calcium), etc.

Table 1 shows the transition temperatures calculated using the formula (1) and taken from the literature.

Further, we assume that the crystal lattice remains the same (we do not change all the coefficients in the formula (1)), but we introduce mercury atoms into the crystal cell - we 
increase the coefficient at $\mathrm{Hg}$ by one, leaving $m$ unchanged:

$$
\frac{1}{T_{c}}=\frac{C_{0} \cdot n \cdot m \cdot(m+1) / 2}{300 \cdot(2 N-3)^{2}}\left(\frac{1}{(m+1) \cdot H g}+\frac{1}{2 \cdot B a}+\frac{1}{(n-1) \cdot C a}+\frac{n}{n \cdot C u+O^{2 n+m+2}}\right) .
$$

In the table 1, the calculation by the formula (2) is shown in the 4th column for (hypothetical) samples with mercury atoms embedded in the crystal lattice. For material with $\mathrm{m}=1$, an increase in $T_{c}$ is visible. Coincidence of $T_{c}=152 \mathrm{~K}$ for Hg-1223, calculated from (2), with the transition temperature of the $\mathrm{Hg}-1223$ sample at a pressure of $P_{0}=150 \mathrm{kbar}$ [10] can be considered not accidental if we assume that the introduction of mercury atoms into the crystal cell of $\mathrm{Hg}-1223$ creates mechanical stresses equivalent to those at a given external pressure $P_{0}$.

Considering the position of the nodes of the crystal lattice of $\mathrm{Hg}-1223$ unchanged upon the introduction of mercury atoms (from one to eight penetrating atoms) into it, we will make an approximate estimate of the magnitude of mechanical stresses in the crystal cell of Hg-1223. Crystal cell parameters of Hg-1223: $a=3.85 \AA$. $c=15.8 \AA$ [11].

Total energy of elastic deformation of the crystal cell [12]:

$$
W=8 \cdot \pi G \cdot r_{0}\left(r_{1}-r_{0}\right)^{2},
$$

where $G=27.6 \mathrm{GPa}$ - shear modulus is taken for another cuprate $\mathrm{Tl}_{2} \mathrm{Ba}_{2} \mathrm{CaCu_{2 } \mathrm { O } _ { 8 + \delta }}$ [13], since for cuprates based on $\mathrm{Hg}$ these modules have not yet been found in the literature; $r_{0}=1 \AA$ - radius of the sphere in the cell where the mercury atom is introduced (preliminary estimate); $r_{1}=1.55 \AA$ is the radius of the mercury atom.

The total energy of elastic deformation of the lattice cell upon the introduction of one mercury atom: $W=2.1 \cdot 10^{-19} \mathrm{~J}$. When eight mercury atoms are introduced into the cell, i.e. as many as in the nodes of cell Hg-1223: $W=16.8 \cdot 10^{-19} \mathrm{~J}$. Then the mechanical stress in the cell in the latter case:

$p=\frac{W}{a \cdot a \cdot c}=\frac{16 \cdot 8 \cdot 10^{-19}}{a \cdot a \cdot c}=80 \mathrm{kbar}$, which coincides in order of magnitude with $P_{0}$.

Thus, it was shown that the incorporation of mercury atoms into the lattice cells of the sample Hg-1223 creates mechanical stresses in the crystal lattice, equivalent to those that arise at increased external pressures, into which a common sample of $\mathrm{Hg}-1223$ is placed.

Further analysis of formula (1) revealed that the combination $300 \cdot 2 / C_{0}$ coincides with the average temperature $T_{c}$ for $\alpha$ - and $\beta$-modifications of mercury: $T_{c}^{H g}=300 \cdot 2 / C_{0}=$ $4.074 K$ [14], [15]. Then formula (1) can be rewritten in the form:

$$
\frac{1}{T_{c}}=\frac{n \cdot m \cdot(m+1)}{T_{c}^{H g} \cdot(2 N-3)^{2}}\left(\frac{1}{m \cdot H g}+\frac{1}{2 \cdot B a}+\frac{1}{(n-1) \cdot C a}+\frac{n}{n \cdot C u+O^{2 n+m+2}}\right),
$$

where the superscript at $T_{c}$ simply denotes the element, but not the power.

Using the same approach as for cuprates, and taking into account the last nuance regarding the nature of the constant $C_{0}$, a formula was obtained that establishes the relationship between the superconducting transition temperature and the material composition of high-temperature superconductors of the family of hydrides $A H_{n}$ or $A D_{n}$, 
where $A=S, L a, Y, \operatorname{Pr}, P, S i, B a, T h$ :

$$
\frac{1}{T_{c}}=\frac{1}{T_{c}^{A} \cdot(2 N+1)}\left(\frac{1}{A}+\frac{h}{n}\right),
$$

where $T_{c}^{A}$ is the critical temperature of the element $A$ (table 2), and the superscript $A$ is just a designation of the element, not an exponent; in brackets $A$ - serial number (nuclear charge) of the element; $n$ - an integer from the material designation - the number of hydrogen or deuterium atoms; $h$ - integer or rational number depending on the space group of the crystal lattice of the sample (table 2); $\mathrm{N}$ is the number of elements in the substance - everywhere $\mathrm{N}=2$, because there are always two elements: $A$ and hydrogen or deuterium.

Table 2 shows the transition temperatures calculated using the formula (5) and taken from the literature. The value of $h$ (in table 2 ) has a specific meaning for each space group and type of crystal lattice - atomic, ionic, metallic or molecular. There are not enough statistics yet to write an exact formula for $h$. In the formula (5), the effect of pressure is taken into account by choosing the value of the critical temperature of component $\mathrm{A}$ at different pressures at which the calculation and experiment coincide best of all (if such $T_{c}^{A}$ are given in the literature). Otherwise, $T_{c}^{A}$ is taken at atmospheric pressure.

In the case of praseodymium, there are no data at ultrahigh pressures of the order of 100 GPa and more. Therefore, Pr can be expected to be a superconductor with a critical temperature of about $2 \mathrm{~K}$ at about $100 \mathrm{GPa}$ or more.

As a next step, based on the previous results, a formula was obtained that establishes the relationship between the superconducting transition temperature and the material composition of high-temperature superconductors of the yttrium-based cuprate family $Y_{m} B a_{k} C u_{n} O_{p}$ :

$$
\frac{1}{T_{c}}=\frac{n_{a} \cdot\left(n_{a}+m\right) \cdot m(m+1)}{T_{c}^{Y} \cdot 2 N}\left(\frac{1}{m \cdot Y}+\frac{1}{k \cdot B a}+\frac{n}{n \cdot C u+O^{p}}\right),
$$

where $T_{c}^{Y}=9.27 \mathrm{~K}$ is the critical temperature for yttrium at $31 \mathrm{GPa}$ [31]; $m, k, n$ and $p$ are integers from the material designation; designation of an element - its serial number, or nuclear charge, i.e. $\mathrm{Y}=39, \mathrm{Ba}=56, \mathrm{Cu}=29, \mathrm{O}=8 ; \mathrm{N}$ is the number of elements, not counting $\mathrm{Cu}-\mathrm{O}$ : i.e. $\mathrm{N}=2 ; n_{a}$ is the number of $\mathrm{Cu}-\mathrm{O}$ planes in the crystal cell [32] (always equal to 2 ).

Table 3 shows the transition temperatures calculated using the formula (6) and taken from the literature.

When outputting (6), other values of $T_{c}^{Y}$ were also used: $T_{c}^{Y}=7.9 \mathrm{~K}$ at $26 \mathrm{GPa}$ [31] and $T_{c}^{Y}=1.5-2.7 \mathrm{~K}$ at 12-16 GPa [33], but the formulas obtained with a different combination of numbers in front of the brackets of (6) gave much worse agreement with the experiment. This suggests that the mechanical stresses in the crystal cell of Y-based cuprates are close to $30 \mathrm{GPa}$, at least near the $\mathrm{Y}$ atom. 


\section{Conclusion}

Using a numerical approach, formulas are obtained that relate the superconducting transition temperature to the composition and structure of the material for various families of high-temperature superconductors. A prediction has been made regarding the method of increasing $T_{c}$ for $\mathrm{Hg}$-based cuprates by implanting $\mathrm{Hg}$ atoms into the crystal lattice of the cuprate, which will create mechanical stresses in the lattice equivalent to stresses at increased external pressure.

It is not surprising that $T_{c}$ would have a systematic dependence on integers, since the consensus view of superconductivity, regardless of physical instantiation, is that of Cooper pairs, formed via the exchange of a boson (phonon). The pairing will depend in a systematic way on the coupling to this boson, the characteristic energy of this boson, and the density of states. All of these quantities reflect the constituent elements in the superconducting compound and, as such, are subject to the systematic variation of elemental properties embedded in the periodic table.

The method has been applied to recently discovered hydrides, and this algorithm agrees with the observed transition temperatures. It is necessary to test this method on other superconductors at high and low pressures to determine its applicability and the underlying laws. The main goal of research is to find a general law and predict a material with the desired $T_{c}$ value at any pressure.

This work has no conflict of interests with anyone.

\section{References}

[1] Plakida N.M. "Microscopic theory of high-temperature superconductivity in strongly correlated electronic systems" Condensed Matter Physics, 2020, vol. 23, No. 4, 43701; https: //doi.org/10.5488/CMP.23.43701

[2] Dale R. Harshman, Anthony T. Fiory and John D. Dow "Theory of high- $T_{C}$ superconductivity: transition temperature" J. Phys.: Condens. Matter 23, 295701 (2011); https://doi .org/10. 1088/0953-8984/23/29/295701

[3] Andrea Benfenati, Andrea Maiani, Filipp N. Rybakov, and Egor Babaev "Vortex nucleation barrier in superconductors beyond the Bean-Livingston approximation: A numerical approach for the sphaleron problem in a gauge theory" Phys. Rev. B 101, 220505(R) (2020); https: //doi.org/10.1103/PhysRevB.101.220505

[4] Covaci L., Peeters F. M., and Berciu M. "Efficient Numerical Approach to Inhomogeneous Superconductivity: The Chebyshev-Bogoliubovde Gennes Method" Phys. Rev. Lett. 105, 167006 (2010); https://doi.org/10.1103/PhysRevLett.105.167006

[5] Balmer J.J. "Notiz ber die Spectrallinien des Wasserstoffs" Annalen der Physik und Chemie, 25, 80 (1885); https://doi.org/10.1002/andp.18852610506

[6] Rydberg J.R. "On the structure of the line-spectra of the chemical elements", Philosophical Magazine, 29, 331 (1890); https://doi.org/10.1080/14786449008619945

[7] Bohr N. "Rydberg's discovery of the spectral laws", Collected works, 10, 373 (1985). 
[8] Roland Hott, Thomas Wolf "Cuprate High Temperature Superconductors" Applied Superconductivity: Handbook on Devices and Applications, Vol. I, Wiley-VCH, Berlin, 152 (2015) arXiv:1502.01557 [cond-mat.supr-con].

[9] David A. Cardwell, David S. Ginley, "Handbook of Superconducting Materials", 1 IoP Publishing Ltd, 2003.

[10] C.W.Chu et. al., "Superconductivity above $150 \mathrm{~K}$ in $\mathrm{HgBa}_{2} \mathrm{Ca}_{2} \mathrm{Cu}_{3} \mathrm{O}_{8+\delta}$ at high pressures", Nature 365, 323 (1993); https://doi.org/10.1038/365323a0

[11] Raivo Stern et. al., "Exploring the oxygen order in Hg-1223", New Challenges in Superconductivity: Experimental Advances and Emerging Theories 69, 183 (2004) arXiv:condmat/0404496 [cond-mat.supr-con] https://doi.org/10.1007/1-4020-3085-1_12

[12] Jacov Frenkel "Kinetic theory of fluids" (in Russian), Nauka, L., 1975.

[13] R. Abd-Shukor, "Ultrasonic and Elastic Properties of Tl- and Hg-Base Cuprate Superconductors: A Review", Phase Transitions 91, 48 (2017) https://doi.org/10.1080/01411594. 2017.1357181.

[14] B. T. Matthiaset. et. al., "Superconductivity", Rev. Mod. Phys. 35, 1 (1963) https://doi. org/10.1103/RevModPhys.35.1.

[15] Julian Eisenstein, "Superconducting Elements", Rev. Mod. Phys. 26, 277 (1954) https: //doi.org/10.1103/RevModPhys.26.277

[16] A. P. Drozdov et. al., "Conventional superconductivity at $203 \mathrm{~K}$ at high pressures", Nature 525, 73 (2015) arXiv:1506.08190 [cond-mat.supr-con]. https://doi.org/10.1038/ nature14964

[17] Russell J. Hemley et. al., "Road to Room-Temperature Superconductivity: Tc above 260 K in Lanthanum Superhydride under Pressure", arXiv:1906.03462 [cond-mat.supr-con].

[18] A. P. Drozdov et. al. "Superconductivity at $250 \mathrm{~K}$ in lanthanum hydride under high pressures", Nature 569, 528 (2019) https://doi.org/10.1038/s41586-019-1201-8; arXiv:1812.01561 [cond-mat.supr-con].

[19] P. P. Kong et. al. "Superconductivity up to $243 \mathrm{~K}$ in yttrium hydrides under high pressure", arXiv:1909.10482 [cond-mat.supr-con].

[20] Di Zhou et. al. "Superconducting Praseodymium Superhydrides", arXiv:1904.06643 [condmat.mtrl-sci].

[21] Hanyu Liu et. al. "Crystal Structure and Superconductivity of PH3 at High Pressures", J. Phys. Chem. C 120, 3458 (2016) https://pubs.acs.org/doi/abs/10.1021/acs.jpcc. 5b12009

[22] A. P. Drozdov, M. I. Eremets, I. A. Troyan "Superconductivity above 100 K in PH3 at high pressures", arXiv:1508.06224 [cond-mat.supr-con].

[23] Jos A. Flores-Livas et. al., "Elemental Phosphorus: structural and superconducting phase diagram under pressure", Phys. Rev. Materials 1, 024802 (2017) https://doi.org/10.1103/ PhysRevMaterials.1.024802

[24] M. I. Eremets et. al. "Superconductivity in Hydrogen Dominant Materials: Silane", Science 319, 1506 (2008) https://doi.org/10.1126/science.1153282

[25] J. M. Mignot, G. Chouteau, G. Martinez "High pressure superconductivity of silicon", Physica B+C 135, 235 (1985) https://doi.org/10.1016/0378-4363(85)90473-5 
[26] Wuhao Chen et. al., "High-Pressure Synthesis of Barium Superhydrides: Pseudocubic BaH12", arXiv:2004.12294 [cond-mat.supr-con].

[27] Dmitry V. Semenok et. al., "Superconductivity at $161 \mathrm{~K}$ in thorium hydride ThH10: Synthesis and properties", Materialstoday 33, 36 (2020) https://doi.org/10.1016/j.mattod.2019. 10.005

[28] Jean-Christophe Griveau, Eric Colineau "Superconductivity in transuranium elements and compounds", C. R. Physique 15, 599 (2014) http://dx.doi.org/10.1016/j.crhy.2014. 07.001

[29] V. V. Struzhkin et. al., "Superconductivity at 10-17 K in compressed sulphur", Nature 390, 382-384 (1997) https://doi.org/10.1038/37074

[30] G. S. Anderson, S. Legvold, and F. H. Spedding "Superconductivity of Lanthanum and Some Lanthanum Alloys", Phys. Rev. 109, 243 (1958); https://doi.org/10.1103/PhysRev. 109. 243

[31] W. Marcin et. al. "High-pressure superconductivity in yttrium: The strong-coupling approach", Solid State Communications 219, 1 (2015) https://doi.org/10.1016/j.ssc. 2015.06.014

[32] Neeraj Khare, "Handbook of High-Temperature Superconductor Electronics", Marcel Dekker Inc., 2003.

[33] Werner Buckel, "Supraleitung", Grundlagen und Anwendungen, Weinheim/Bergstr. 1972. 


\begin{tabular}{|c|c|c|c|c|c|}
\hline $\begin{array}{c}\text { Composition: } \\
A H_{n}\end{array}$ & $\begin{array}{l}\text { space } \\
\text { group }\end{array}$ & $\begin{array}{c}\text { experimental } \\
T_{c}, \text { [source] }\end{array}$ & $\begin{array}{c}T_{c} \\
\text { by the formula }(5)\end{array}$ & $\begin{array}{c}T_{c}^{A} \\
\text { [source] }\end{array}$ & $h$ \\
\hline $\mathrm{H}_{3} \mathrm{~S}$ & $I m-3 m$ & $\begin{array}{c}203.5 \mathrm{~K} \\
\text { at } 155 \mathrm{GPa}[16\end{array}$ & $202.1 K$ & $\begin{array}{c}T_{c}^{S}=16 \mathrm{~K} \\
\text { at } 160 \mathrm{GPa}[29\end{array}$ & 1 \\
\hline$D_{2} S$ & $I m-3 m$ & $\begin{array}{c}150 \mathrm{~K} \\
\text { at } 155 \mathrm{GPa}[16]\end{array}$ & $142 K$ & $\begin{array}{c}T_{c}^{S}=16 K \\
\text { at } 160 \mathrm{GPa}[29]\end{array}$ & 1 \\
\hline $\mathrm{LaH}_{10}$ & $F m \overline{3} m$ & $\begin{array}{c}260 \mathrm{~K} \\
\text { at } 200 \mathrm{GPa}[17\end{array}$ & $253 K$ & $\begin{array}{l}T_{c}^{L a}=5.95 \mathrm{~K} \\
\text { at ambient pressure } 30]\end{array}$ & 1 \\
\hline$L a D_{11}$ & $\mathrm{P} 4 / \mathrm{nmm}$ & $\begin{array}{c}140 \mathrm{~K} \\
\text { at } 142 \mathrm{GPa}[18\end{array}$ & $149 K$ & $\begin{array}{c}T_{c}^{L a}=5.95 \mathrm{~K} \\
\text { at ambient pressure [30] }\end{array}$ & 2 \\
\hline$Y H_{6}$ & $I m-3 m$ & $\begin{array}{c}227 \mathrm{~K} \\
\text { at } 237 \mathrm{GPa}[19\end{array}$ & $241 K$ & $\begin{array}{l}T_{c}^{Y}=9.27 \mathrm{~K} \\
\text { at } 31 \mathrm{GPa}[31]\end{array}$ & 1 \\
\hline$Y_{9}$ & $P 6_{3} / m m c$ & $\begin{array}{c}243 \mathrm{~K} \\
\text { at } 201 \mathrm{GPa}[19]\end{array}$ & $241 K$ & $\begin{array}{c}T_{c}^{Y}=9.27 \mathrm{~K} \\
\text { at } 31 \mathrm{GPa}[31]\end{array}$ & $6 / 4$ \\
\hline $\mathrm{PrH}_{9}$ & $P 6_{3} / m m c$ & $\begin{array}{c}55 \mathrm{~K} \\
\text { at }>110 \mathrm{GPa}[20]\end{array}$ & $54.4 K$ & $T_{c}^{P r}=2 K$ & $6 / 4$ \\
\hline $\mathrm{PrH}_{9}$ & $F \overline{4} 3 m$ & $\begin{array}{c}69 \mathrm{~K} \\
\text { at } 120 \mathrm{GPa}[20]\end{array}$ & $61 K$ & $T_{c}^{P r}=2 K$ & $4 / 3$ \\
\hline $\mathrm{PH}_{3}$ & $\begin{array}{l}C 2 / m \\
{[21]}\end{array}$ & $\begin{array}{c}103 \mathrm{~K} \\
\text { at } 207 \mathrm{GPa}[22]\end{array}$ & $112 \mathrm{~K}$ & $\begin{array}{c}T_{c}^{P}=6.5 K \\
\text { at } 200 \mathrm{GPa}[23]\end{array}$ & $2 / 3$ \\
\hline $\mathrm{SiH}_{4}$ & $P 6_{3}$ & $\begin{array}{c}17 K \\
\text { at } 96 \text { and } \\
120 \mathrm{GPa}[24\end{array}$ & $16.8 K$ & $\begin{array}{c}T_{c}^{S i}=3.6 K \\
\text { at } 25 \mathrm{GPa}[25]\end{array}$ & 4 \\
\hline $\mathrm{BaH}_{12}$ & $C m c 2_{1}$ & $\begin{array}{c}20 \mathrm{~K} \\
\text { at } 140 \mathrm{GPa}[26]\end{array}$ & $21 K$ & $\begin{array}{c}T_{c}^{B a}=5 K \\
\text { at } 18 \mathrm{GPa}[26]\end{array}$ & 14 \\
\hline $\mathrm{ThH}_{9}$ & $P 6_{3} / m m c$ & $\begin{array}{c}146 \mathrm{~K} \\
\text { at } 170 \mathrm{GPa} \text { [27] }\end{array}$ & $142.7 \mathrm{~K}$ & $\begin{array}{l}T_{c}^{T h}=1.374 K \\
\text { at ambient [28] }\end{array}$ & $1 / 3$ \\
\hline $\mathrm{ThH}_{10}$ & $F m \overline{3} m$ & $\begin{array}{c}160 \mathrm{~K} \\
\text { at } 170 \mathrm{GPa}[27]\end{array}$ & $154.6 K$ & $\begin{array}{l}T_{c}^{T h}=1.374 K \\
\text { at ambient }[28]\end{array}$ & $1 / 3$ \\
\hline
\end{tabular}

Table 2: Table of experimental values of the critical temperature of hydrides in comparison with that calculated by the formula (5).

\begin{tabular}{|c|c|c|}
\hline $\begin{array}{l}\text { Composition: } \\
Y_{m} B a_{k} C u_{n} O_{p}\end{array}$ & $\begin{array}{c}\text { experimental } \\
T_{c}, \text { [source] }\end{array}$ & $\begin{array}{c}T_{c} \\
\text { by the formula }(6)\end{array}$ \\
\hline $\mathrm{YBa}_{2} \mathrm{Cu}_{3} \mathrm{O}_{7}$ & $92 K \quad[32]$ & $89 K$ \\
\hline $\mathrm{YBa}_{2} \mathrm{Cu}_{4} \mathrm{O}_{8}$ & $80 K[32]$ & $89 K$ \\
\hline $\mathrm{Y}_{2} \mathrm{Ba}_{4} \mathrm{Cu}_{7} \mathrm{O}_{14}$ & $40 K[32]$ & $45 K$ \\
\hline
\end{tabular}

Table 3: Table of experimental values of the critical temperature of Y-based cuprates in comparison with that calculated by formula (6). 